\title{
CHARACTERIZATION OF WEIGHTED BESOV SPACES
}

\author{
José Luis Ansorena And Oscar Blasco
}

\begin{abstract}
We find conditions on the weight $w$ in order characterize functions in weighted Besov spaces $B_{w, \phi}^{p, q}$ in terms of differences $\Delta_{x} f$.
\end{abstract}

\section{INTRODUCTION.}

There are many ways to define Besov spaces (see $[1,19,24]$ ). It is well known that Besov spaces can be defined, for instance in terms of convolutions $f * \phi_{t}$ with different kinds of smooth functions $\phi$ and that they can be also described by means of differences $\Delta_{x} f($ see $[10,11,22])$.

Our objective will be to find weights (which extend the case $t^{\alpha}$ ) where we can still get such a characteritation of weighted Besov spaces and to give a general procedure which works not only in the classical case but also in the weighted one. Our arguments will be based upon two main points: The Calderón's formula, a quite simple Schur Lemma.

We want to notice that this characterization can be used to get the atomic decomposition of the spaces.

The paper is divided into two sections. Section 1 has a preliminary character and it is devoted to introduce the notation and the main lemmas to be used later on. In Section 2 we prove the result about coincidence of seminorms in the spaces defined by differences and convolutions.

Throughout the paper a weight $w: \mathbb{R}^{+} \rightarrow \mathbb{R}^{+}$will be a measurable function $w>0$ a.e., $1 \leq p, q \leq \infty$ and $p^{\prime}, q^{\prime}$ stand for the conjugate exponents. $\mathcal{S}$ denotes the Schwartz class of test functions on $\mathbb{R}^{n}, \mathcal{S}^{\prime}$ the space of tempered distributions, $\mathcal{S}_{0}$ the set of functions in $\mathcal{S}$ with mean zero and $\mathcal{S}^{\prime}{ }_{0}$ its topological dual.

Given a weight $w$ and $1 \leq p, q \leq \infty$ we shall denote by $\Lambda_{w}^{p, q}$ the space of measurable functions $f: \mathbb{R}^{n} \rightarrow \mathbb{C}$ such that

$$
\|f\|_{\Lambda_{w}^{p, q}}=\left(\int_{\mathbb{R}^{n}} \frac{\left\|\Delta_{x} f\right\|_{p}^{q}}{w(|x|)^{q}} \frac{d x}{|x|^{n}}\right)^{\frac{1}{q}}<\infty \quad(1 \leq q<\infty)
$$

or

1991 Mathematics Subject Classification. 42A45, 42B25, 42C15.

Key words and phrases. Besov spaces, weights.

The authors has been partially supported by the Spanish DGICYT, Proyecto PS89-0106 and PB92-0699

Typeset by $\mathcal{A} \mathcal{M} \mathcal{S}-\mathrm{T}_{\mathrm{E}} \mathrm{X}$ 


$$
\|f\|_{\Lambda_{w}^{p, \infty}}=\inf \left\{C>0:\left\|\Delta_{x} f\right\|_{p} \leq C w(|x|) \text { a.e.x } \in \mathbb{R}^{n}\right\}<\infty \quad(q=\infty)
$$

where $\Delta_{x} f(y)=f(x+y)-f(y)$.

Given a weight $w, \phi \in \mathcal{S}_{0}$ and $1 \leq p, q \leq \infty$ we shall denote by $B_{w, \phi}^{p, q}$ the space functions $f: \mathbb{R}^{n} \rightarrow \mathbb{C}$ with $f \in L^{1}\left(\mathbb{R}^{n}, \frac{d x}{(1+|x|)^{n+1}}\right)$ such that

$$
\|f\|_{B_{w, \phi}^{p, q}}=\left(\int_{\mathbb{R}^{n}} \frac{\left\|\phi_{t} * f\right\|_{p}^{q}}{w(t)^{q}} \frac{d t}{t}\right)^{\frac{1}{q}}<\infty \quad(1 \leq q<\infty)
$$

or

$$
\|f\|_{B_{w, \phi}^{p, \infty}}=\inf \left\{C>0:\left\|\phi_{t} * f\right\|_{p} \leq C w(t) \quad \text { a.e.t }>0\right\}<\infty \quad(q=\infty)
$$

where $\phi_{t}(x)=\frac{1}{t^{n}} \phi\left(\frac{x}{t}\right)$.

To state the results of the paper, let us first recall the following notions.

A weight $w$ is said to satisfy Dini condition if there exists $C>0$ such that

$$
\int_{0}^{s} \frac{w(t)}{t} d t \leq C w(s) \text { a.e. } s>0 .
$$

A weight $w$ is said to be a $b_{1}$-weight if there exists $C>0$ such that

$$
\int_{s}^{\infty} \frac{w(t)}{t^{2}} d t \leq C \frac{w(s)}{s} \text { a.e. } s>0
$$

We shall denote by $\mathcal{W}_{0,1}$ the space of $b_{1}$-weights which satisfy Dini condition.

Let us also use the notation $\mathcal{A}$ and $\mathcal{A}_{1}$ for the following classes

$$
\mathcal{A}=\left\{\phi \in \mathcal{S}_{0}: \int_{0}^{\infty}(\hat{\phi}(t \xi))^{2} \frac{d t}{t}=1 \text { for } \xi \in \mathbb{R}^{n} \backslash\{0\}\right\} .
$$

$\mathcal{A}_{1}=\left\{\phi \in \mathcal{A}: \phi\right.$ radial and real, supp $\left.\phi \subseteq\{|x| \leq 1\}, \int_{\mathbb{R}^{n}} x_{i} \phi(x) d x=0, i=1, \ldots, n\right\}$.

Section 2 is devoted to prove the following theorem.

Main Theorem. Let $1 \leq p, q \leq \infty, \phi \in \mathcal{A}$ and $w$ be a weight that can be factorized as $w(t)=\lambda^{\frac{1}{q^{\prime}}}(t) \mu^{\frac{-1}{q}}\left(t^{-1}\right)$ where $\lambda, \mu \in \mathcal{W}_{0,1}$. Then

$$
\Lambda_{w}^{p, q}=B_{w, \phi}^{p, q} \quad \text { (with equivalent seminorms). }
$$

For particular cases $w(t)=t^{\alpha}$ the reader is referred to $[10,11,14,22]$ for similar results for special functions $\phi$ and their applications. In our weighted situation some closely related results for the unit disc are included in [3] and [5].

The reader should be aware that the case $1<q<\infty$ in Main Theorem could have been shown by interpolation with the extreme cases, but a direct proof is presented in the paper. 


\section{$\S 1 . \quad$ PRELIMINARIES.}

Let us recall some notions on weights we shall need later.

Definition 1.1. Let $\varepsilon \geq 0, \delta \geq 0$ and $w$ be a weight. $w$ is said to be a $d_{\varepsilon}$-weight if exists $C>0$ such that

$$
\int_{0}^{s} t^{\varepsilon} w(t) \frac{d t}{t} \leq C s^{\varepsilon} w(s) \text { a.e. } s>0 .
$$

$w$ is said to be a $b_{\delta}$-weight if there exists $C>0$ such that

$$
\int_{s}^{\infty} \frac{w(t)}{t^{\delta}} \frac{d t}{t} \leq C \frac{w(s)}{s^{\delta}} \text { a.e. } s>0
$$

If $\left(d_{\varepsilon}\right)$ (respect. $\left.\left(b_{\delta}\right)\right)$ denotes de class of $d_{\varepsilon}$-weights (respect. $b_{\delta}$-weights) we write

$$
\mathcal{W}_{\varepsilon, \delta}=\left(d_{\varepsilon}\right) \cap\left(b_{\delta}\right)
$$

The following properties are elementary and left to the interested reader

$$
\begin{gathered}
w \in\left(d_{\varepsilon}\right) \Rightarrow w \in\left(d_{\varepsilon^{\prime}}\right) \text { for any } \varepsilon^{\prime}>\varepsilon . \\
w \in\left(b_{\delta}\right) \Rightarrow w \in\left(b_{\delta^{\prime}}\right) \text { for any } \delta^{\prime}>\delta . \\
\text { Let } \bar{w}(t)=w\left(t^{-1}\right) \text { then } w \in\left(b_{\varepsilon}\right) \Longleftrightarrow \bar{w} \in\left(d_{\varepsilon}\right) . \\
w \in \mathcal{W}_{\varepsilon, \delta} \Rightarrow w(t) \geq C \min \left(t^{-\varepsilon}, t^{\delta}\right) .
\end{gathered}
$$

Let us now give some examples.

It is elementary to see that if $\alpha \in \mathbb{R}$ and $w_{\alpha}(t)=t^{\alpha}$ then $w_{\alpha} \in \mathcal{W}_{\varepsilon, \delta}$ for any $\delta>\alpha$ and $\varepsilon>-\alpha$.

Let us give a bit more general example. Let $\alpha, \beta \in \mathbb{R}$ and $w_{\alpha, \beta}(t)=t^{\alpha}(1+|\log t|)^{\beta}$. Then $w_{\alpha, \beta} \in \mathcal{W}_{\varepsilon, \delta}$ for any $\delta>\alpha$ and $\varepsilon>-\alpha$.

Indeed, let us take $\delta>\alpha$. Then making the change of variable $t=s u$ we have

$$
\begin{aligned}
\int_{s}^{\infty} \frac{w_{\alpha, \beta}(t)}{t^{\delta+1}} d t & =\int_{s}^{\infty} t^{\alpha-\delta}(1+|\log t|)^{\beta} \frac{d t}{t} \\
& \leq s^{\alpha-\delta} \int_{1}^{\infty} u^{\alpha-\delta}(1+|\log s|+\log u)^{\beta} \frac{d u}{u} .
\end{aligned}
$$


For $\beta<0$ then

$$
\int_{s}^{\infty} \frac{w_{\alpha, \beta}(t)}{t^{\delta+1}} d t \leq \frac{1}{\delta-\alpha} s^{\alpha-\delta}(1+|\log s|)^{\beta}=\frac{C_{\beta}}{\delta-\alpha} \frac{w_{\alpha, \beta}(s)}{s^{\delta}}
$$

For $\beta>0$, using $(a+b)^{\beta} \leq C_{\beta}\left(a^{\beta}+b^{\beta}\right)$, we have

$$
\begin{aligned}
\int_{s}^{\infty} \frac{w_{\alpha, \beta}(t)}{t^{\delta+1}} d t & \leq C_{\beta} s^{\alpha-\delta}\left((1+|\log s|)^{\beta} \int_{1}^{\infty} u^{\alpha-\delta} \frac{d u}{u}+\int_{1}^{\infty} u^{\alpha-\delta}(\log u)^{\beta} \frac{d u}{u}\right) \\
& \leq C(\alpha, \beta, \delta) \frac{w_{\alpha, \beta}(s)}{s^{\delta}} .
\end{aligned}
$$

Since $w_{\alpha, \beta}(t)=w_{-\alpha, \beta}\left(t^{-1}\right)$ then also have $w_{\alpha, \beta}$ is a $d_{\varepsilon^{-}}$weight for $\varepsilon>-\alpha$.

Let us now establish the main lemma to be used later on. Observe that a net $\left\{\phi_{i}\right\}_{i \in \Lambda}$ converges to $\phi$ in $\mathcal{S}_{0}^{\prime}$ if there exist $\left\{c_{i}\right\}_{i \in \Lambda} \subset \mathbb{C}$ such that $\phi_{i}-c_{i}$ converges to $\phi$ in $\mathcal{S}^{\prime}$.

One of the main facts in our approach, which follows ideas from [6] and [14], is the use of the Calderón reproducing formula.

Let $\phi \in \mathcal{A}$ and $\psi \in \mathcal{S}$ then for $\xi \in \mathbb{R}^{n} \backslash\{0\}$,

$$
\hat{\psi}(\xi)=\int_{0}^{\infty}\left(\phi_{t} * \phi_{t} * \psi\right) \hat{(\xi)} \frac{d t}{t}
$$

This shows that $\psi_{\varepsilon, \delta}=\int_{\varepsilon}^{\delta} \phi_{t} * \phi_{t} * \psi \frac{d t}{t}$ converges to $\psi$ in $\mathcal{S}$.

Lemma A. (see Appendix [14]). Let $f \in L^{1}\left(\mathbb{R}^{n}, \frac{d x}{(1+|x|)^{n+1}}\right)$ and $\phi \in \mathcal{A}$. For $0<\varepsilon<\delta$ define

$$
f_{\varepsilon, \delta}(x)=\int_{\varepsilon}^{\delta}\left(\phi_{t} * \phi_{t} * f\right)(x) \frac{d t}{t}
$$

Then $f_{\varepsilon, \delta}$ converges to $f$ in $\mathcal{S}^{\prime}{ }_{0}$ as $\varepsilon \rightarrow 0$ and $\delta \rightarrow \infty$.

To finish this preliminary section let us state a version of Schur lemma that will be useful for our purposes and whose elementary proof we include here for the sake of completeness.

Lemma B. Let $1<q<\infty$ and $\frac{1}{q}+\frac{1}{q^{\prime}}=1$. Let $\left(\Omega_{1}, \Sigma_{1}, \mu_{1}\right)$ and $\left(\Omega_{2}, \Sigma_{2}, \mu_{2}\right)$ be two $\sigma$-finite measure spaces and let $K: \Omega_{1} \times \Omega_{2} \rightarrow \mathbb{R}^{+}$be a measurable function and write $T_{K}(f)$ for

$$
T_{K}(f)\left(w_{2}\right)=\int_{\Omega_{1}} K\left(w_{1}, w_{2}\right) f\left(w_{1}\right) d \mu_{1}\left(w_{1}\right) .
$$

If there exist $C>0$ and measurable functions $h_{i}: \Omega_{i} \rightarrow \mathbb{R}^{+}(i=1,2)$ such that

$$
\int_{\Omega_{1}} K\left(w_{1}, w_{2}\right) h_{1}^{q^{\prime}}\left(w_{1}\right) d \mu_{1}\left(w_{1}\right) \leq C h_{2}^{q^{\prime}}\left(w_{2}\right) \quad \mu_{2}-\text { a.e. }
$$




$$
\int_{\Omega_{2}} K\left(w_{1}, w_{2}\right) h_{2}^{q}\left(w_{2}\right) d \mu_{2}\left(w_{2}\right) \leq C h_{1}^{q}\left(w_{1}\right) \mu_{1}-\text { a.e. }
$$

Then $T_{K}$ defines a bounded operator from $L^{q}\left(\Omega_{1}, \mu_{1}\right)$ into $L^{q}\left(\Omega_{2}, \mu_{2}\right)$.

Proof. From (1.9) and Hölder's inequality we have

$$
\left|T_{K}(f)\left(w_{2}\right)\right| \leq C h_{2}\left(w_{2}\right)\left(\int_{\Omega_{1}} K\left(w_{1}, w_{2}\right) h_{1}^{-q}\left(w_{1}\right)\left|f\left(w_{1}\right)\right|^{q} d \mu_{1}\left(w_{1}\right)\right)^{\frac{1}{q}} .
$$

Apply now (1.10) and Fubini's theorem to get

$$
\begin{aligned}
\left\|T_{K}(f)\right\|_{q} & \leq C\left(\int_{\Omega_{1}}\left(\int_{\left.\Omega_{2}\right)} K\left(w_{1}, w_{2}\right) h_{2}^{q}\left(w_{2}\right) d \mu_{2}\left(w_{2}\right)\right) h_{1}\left(w_{1}\right)^{-q}\left|f\left(w_{1}\right)\right|^{q} d \mu_{1}\left(w_{1}\right)\right)^{\frac{1}{q}} \\
& \leq C^{2}\|f\|_{q}
\end{aligned}
$$

\section{§2. Characterization of Besov spaces}

Let us first establish some general facts that can be used to relate properties about differences $\Delta_{x} f$ and convolutions $\phi_{t} * f$.

Lemma 2.1. Let $1 \leq p \leq \infty, \rho \geq 0$ and $\phi \in \mathcal{A}$. Then there exists $C>0$ such that if $f \in L^{1}\left(\mathbb{R}^{n}, \frac{d x}{(1+|x|)^{n+1}}\right)$ then we have:

$$
\left\|\phi_{t} * f\right\|_{p} \leq C \int_{\mathbb{R}^{n}} \min \left(\left(\frac{|x|}{t}\right)^{n},\left(\frac{t}{|x|}\right)^{\rho}\right)\left\|\Delta_{x} f\right\|_{p} \frac{d x}{|x|^{n}}
$$

$$
\left\|\Delta_{x} f\right\|_{p} \leq C \int_{0}^{\infty} \min \left(1, \frac{|x|}{t}\right)\left\|\phi_{t} * f\right\|_{p} \frac{d t}{t}
$$

Proof. Notice that, since $\int_{\mathbb{R}^{n}} \phi(x) d x=0$, then

$$
\phi_{t} * f(y)=\int_{\mathbb{R}^{n}} \phi_{t}(x) \Delta_{-x} f(y) d x .
$$

From Minkowski's inequality one gets

$$
\left\|\phi_{t} * f\right\|_{p} \leq \int_{\mathbb{R}^{n}} \frac{|x|^{n}}{t^{n}}\left|\phi\left(\frac{-x}{t}\right)\right|\left\|\Delta_{x} f\right\|_{p} \frac{d x}{|x|^{n}}
$$

Hence (2.1) follows from the trivial estimates 


$$
\begin{aligned}
|y|^{n+\rho}|\phi(y)| \leq C & \text { if } \quad|y| \geq 1 \\
|\phi(y)| \leq C & \text { if } \quad|y| \leq 1
\end{aligned}
$$

To prove (2.2) observe first that for $0<\varepsilon<\delta$

$$
\Delta_{x} f_{\varepsilon, \delta}(y)=\int_{\varepsilon}^{\delta}\left(\Delta_{-x} \phi_{t}\right) * \phi_{t} * f(y) \frac{d t}{t}
$$

Hence Minkowski's inequality and Young's inequality give

$$
\left\|\Delta_{x} f_{\varepsilon, \delta}\right\|_{p} \leq \int_{\varepsilon}^{\delta}\left\|\Delta_{-x} \phi_{t}\right\|_{1}\left\|\phi_{t} * f\right\|_{p} \frac{d t}{t} .
$$

Note that

$$
\begin{aligned}
& \left\|\Delta_{y} \phi\right\|_{1} \leq 2\|\phi\|_{1} \quad \text { if } \quad|y| \geq 1 . \\
& \left\|\Delta_{y} \phi\right\|_{1} \leq|y| \int_{\mathbb{R}^{n}} \max _{|z-u|<1}|\nabla \phi(z)| d u \quad \text { if } \quad|y| \leq 1 .
\end{aligned}
$$

Hence

$$
\left\|\Delta_{-x} \phi_{t}\right\|_{1}=\left\|\Delta_{\frac{-x}{t}} \phi\right\|_{1} \leq C \min \left(1, \frac{|x|}{t}\right) .
$$

Therefore, using the previous estimate $\left(2.2^{\prime}\right)$ and Lemma A, a simple limiting argument shows (2.2).

Although for the purposes of this paper only a particular case of next lemma will be used we state a general version of it that we find interesting in its own right.

Lemma 2.2. Given $0 \leq \varepsilon, \delta<\infty, 1<q<\infty$, and $w$ a weight, let us consider

$$
R_{\varepsilon, \delta}(s, t)=\frac{w(s)}{w(t)} \min \left(\left(\frac{s}{t}\right)^{\varepsilon},\left(\frac{t}{s}\right)^{\delta}\right) .
$$

If $w(s)=\lambda^{\frac{1}{q^{\prime}}}(s) \mu^{\frac{-1}{q}}\left(s^{-1}\right)$ for some pair of weights $\lambda, \mu \in \mathcal{W}_{\varepsilon, \delta}$, then there exist $C>0$ and $g: \mathbb{R}^{+} \rightarrow \mathbb{R}^{+}$measurable such that

$$
\begin{gathered}
\int_{0}^{\infty} R_{\varepsilon, \delta}(s, t) g^{q^{\prime}}(s) \frac{d s}{s} \leq C g^{q^{\prime}}(t) . \\
\int_{0}^{\infty} R_{\varepsilon, \delta}(s, t) g^{q}(t) \frac{d t}{t} \leq C g^{q}(s) .
\end{gathered}
$$

Proof. Let us take $g(t)=\lambda^{\frac{1}{q q^{\prime}}}(t) \mu^{\frac{1}{q q^{\prime}}}\left(t^{-1}\right)$. Then $g^{q^{\prime}}(s)=\frac{\lambda(s)}{w(s)}$ and $g^{q}(t)=w(t) \mu\left(t^{-1}\right)$. 
Therefore

$$
\begin{aligned}
\int_{0}^{\infty} R_{\varepsilon, \delta}(s, t) g^{q^{\prime}}(s) \frac{d s}{s} & =\frac{1}{w(t)} \int_{0}^{\infty} \lambda(s) \min \left(\left(\frac{s}{t}\right)^{\varepsilon},\left(\frac{t}{s}\right)^{\delta}\right) \frac{d s}{s} \\
& =\frac{1}{t^{\varepsilon} w(t)} \int_{0}^{t} s^{\varepsilon} \lambda(s) \frac{d s}{s}+\frac{t^{\delta}}{w(t)} \int_{t}^{\infty} \frac{\lambda(s)}{s^{\delta}} \frac{d s}{s} \\
& \leq C \frac{\lambda(t)}{w(t)}=C g^{q^{\prime}}(t) .
\end{aligned}
$$

On the other hand

$$
\begin{aligned}
\int_{0}^{\infty} R_{\varepsilon, \delta}(s, t) g^{q}(t) \frac{d t}{t} & =w(s) \int_{0}^{\infty} \mu\left(t^{-1}\right) \min \left(\left(\frac{s}{t}\right)^{\varepsilon},\left(\frac{t}{s}\right)^{\delta}\right) \frac{d t}{t} \\
& =\frac{w(s)}{s^{\delta}} \int_{0}^{s} t^{\delta} \mu\left(t^{-1}\right) \frac{d}{t}+s^{\varepsilon} w(s) \int_{s}^{\infty} \frac{\mu\left(t^{-1}\right)}{t^{\varepsilon}} \frac{d t}{t} \\
& =\frac{w(s)}{s^{\delta}} \int_{s^{-1}}^{\infty} \frac{\mu(t)}{t^{\delta}} \frac{d}{t}+s^{\varepsilon} w(s) \int_{0}^{s^{-1}} t^{\varepsilon} \mu(t) \frac{d t}{t} \\
& \leq C \mu\left(s^{-1}\right) w(s)=C g^{q}(s) .
\end{aligned}
$$

Let us now state the following result in order to avoid repeating arguments in several of the remaining proofs.

Lemma 2.3. Let $1 \leq p \leq \infty$ and let $f$ be a measurable function.

If $\left\|\Delta_{x} f\right\|_{p} \in L^{1}\left(\mathbb{R}^{n}, \frac{d x}{(1+|x|)^{n+1}}\right)$ then $f \in L^{1}\left(\mathbb{R}^{n},\left(\frac{d x}{1+|x|)^{n+1}}\right)\right.$.

Proof. Choose $\Psi \in L^{p^{\prime}}\left(\mathbb{R}^{n}, d x\right)$ with $\Psi>0$ a.e. Then Hölder's inequality and Fubini's theorem give

$$
\int_{\mathbb{R}^{n}}\left(\int_{\mathbb{R}^{n}} \frac{|f(x+y)-f(y)|}{(1+|x|)^{n+1}} d x\right) \Psi(y) d y<\infty .
$$

Therefore

$$
\int_{\mathbb{R}^{n}} \frac{|f(x+y)-f(y)|}{(1+|x|)^{n+1}} d x<\infty \text { for a.e. } y \in \mathbb{R}^{n} .
$$

Since $(1+|x|)^{-(n+1)} \in L^{1}\left(\mathbb{R}^{n}\right)$ then

$$
\int_{\mathbb{R}^{n}} \frac{|f(x+y)|}{(1+|x|)^{n+1}} d x<\infty \text { for a.e. } y \in \mathbb{R}^{n} .
$$

Finally since there exists $C>0$ such that $1+|x+y| \geq C(1+|x|)$ for all $y \in \mathbb{R}^{n}$, then one has 


$$
\int_{\mathbb{R}^{n}} \frac{|f(x)|}{(1+|x|)^{n+1}} d x<\infty .
$$

Let us now start with the case $q=\infty$ in the Main Theorem which easily follows from Lemma 2.1.

Theorem 2.1. Let $1 \leq p \leq \infty, \phi \in \mathcal{A}$ and $w \in \mathcal{W}_{0,1}$. Then

$$
\Lambda_{w}^{p, \infty}=B_{w, \phi}^{p, \infty} \quad \text { (with equivalent seminorms) }
$$

Proof. Assume $f \in \Lambda_{w}^{p, \infty}$. Note that

$$
\begin{aligned}
\int_{\mathbb{R}^{n}} \frac{\left\|\Delta_{x} f\right\|_{p}}{(1+|x|)^{n+1}} d x & \leq C \int_{\mathbb{R}^{n}} \frac{w(|x|)}{(1+|x|)^{n+1}} d x \\
& \leq C \int_{0}^{\infty} \frac{w(t) t^{n-1}}{(1+t)^{n+1}} d t \\
& \leq C\left(\int_{0}^{1} w(t) \frac{d t}{t}+\int_{1}^{\infty} w(t) \frac{d t}{t^{2}}\right)<\infty
\end{aligned}
$$

what combined with Lemma 2.3 gives

$$
\int_{\mathbb{R}^{n}} \frac{|f(x)|}{(1+|x|)^{n+1}} d x<\infty .
$$

Let us prove that $\left\|\phi_{t} * f\right\|_{p} \leq C w(t)$. From (2.1) in Lemma 2.1 for $\rho=1$ we have

$$
\begin{aligned}
\left\|\phi_{t} * f\right\|_{p} & \leq C\left(\frac{1}{t^{n}} \int_{|x|<t}\left\|\Delta_{x} f\right\|_{p} d x+t \int_{|x|>t}\left\|\Delta_{x} f\right\|_{p} \frac{d x}{|x|^{n+1}}\right) \\
& \leq C\left(\frac{1}{t^{n}} \int_{|x|<t} w(|x|) d x+t \int_{|x|>t} w(|x|) \frac{d x}{|x|^{n+1}}\right) \\
& \leq C\left(\int_{0}^{t}\left(\frac{s}{t}\right)^{n} w(s) \frac{d s}{s}+t \int_{t}^{\infty} w(s) \frac{d s}{s^{2}}\right) \leq C w(t) .
\end{aligned}
$$

Assume now $f \in B_{w, \phi}^{p, \infty}$. Then from (2.2) we have

$$
\begin{aligned}
\left\|\Delta_{x} f\right\|_{p} & \leq C\left(\int_{0}^{|x|}\|\phi * f\|_{p} \frac{d t}{t}+|x| \int_{|x|}^{\infty}\|\phi * f\|_{p} \frac{d t}{t^{2}}\right) \\
& \leq C\left(\int_{0}^{|x|} \frac{w(t)}{t} d t+|x| \int_{|x|}^{\infty} \frac{w(t)}{t^{2}} d t\right) \leq C w(|x|) .
\end{aligned}
$$

We prove now the case $q=1$ in the Main Theorem. 
Theorem 2.2. Let $1 \leq p \leq \infty, \phi \in \mathcal{A}$ and $w$ such that $\mu(t)=w^{-1}\left(t^{-1}\right) \in \mathcal{W}_{0,1}$. Then

$$
\Lambda_{w}^{p, 1}=B_{w, \phi}^{p, 1} \quad \text { (with equivalent seminorms). }
$$

Proof. Assume $f \in \Lambda_{w}^{p, 1}$. Let us first prove that

$$
\int_{\mathbb{R}^{n}} \frac{|f(x)|}{(1+|x|)^{n+1}} d x<\infty
$$

From (1.5)

$$
\frac{1}{|x|^{n} w(|x|)} \geq C \frac{1}{|x|^{n}} \min \left(1, \frac{1}{|x|}\right) \geq C \frac{1}{|x|^{n}} \min \left(|x|^{n}, \frac{1}{|x|}\right) \geq \frac{C}{(1+|x|)^{n+1}}
$$

Hence

$$
\int_{\mathbb{R}^{n}} \frac{\left\|\Delta_{x} f\right\|_{p}}{(1+|x|)^{n+1}} d x \leq C \int_{\mathbb{R}^{n}} \frac{\left\|\Delta_{x} f\right\|_{p}}{w(|x|)} \frac{d x}{|x|^{n}}<\infty
$$

and we apply Lemma 2.3 again.

We shall now prove that $\|f\|_{B_{w, \phi}^{p, 1}} \leq C\|f\|_{\Lambda_{w}^{p, 1}}$.

Using (2.1) in Lemma 2.1 with $\rho=1$

$$
\begin{aligned}
\int_{0}^{\infty} \frac{\left\|\phi_{t} * f\right\|_{p}}{w(t)} \frac{d t}{t} & \leq C \int_{0}^{\infty}\left[\int_{\mathbb{R}^{n}} \min \left(\left(\frac{|x|}{t}\right)^{n},\left(\frac{t}{|x|}\right)\right) \frac{\left\|\Delta_{x} f\right\|_{p}}{w(t)} \frac{d x}{|x|^{n}}\right] \frac{d t}{t} \\
& =C \int_{\mathbb{R}^{n}}\left\|\Delta_{x} f\right\|_{p}\left[\int_{0}^{\infty} \min \left(\left(\frac{|x|}{t}\right)^{n},\left(\frac{t}{|x|}\right)\right) \mu\left(t^{-1}\right) \frac{d t}{t}\right] \frac{d x}{|x|^{n}} \\
& =C \int_{\mathbb{R}^{n}}\left\|\Delta_{x} f\right\|_{p}\left[\int_{0}^{|x|} \frac{t \mu\left(t^{-1}\right)}{|x|} \frac{d t}{t}+\int_{|x|}^{\infty} \frac{|x|^{n} \mu\left(t^{-1}\right)}{t^{n}} \frac{d t}{t}\right] \frac{d x}{|x|^{n}} \\
& \leq C \int_{\mathbb{R}^{n}}\left\|\Delta_{x} f\right\|_{p}\left[\frac{1}{|x|} \int_{|x|^{-1}}^{\infty} \mu(t) \frac{d t}{t^{2}}+\int_{0}^{|x|^{-1}} \mu(t) \frac{d t}{t}\right] \frac{d x}{|x|^{n}} \\
& \leq C \int_{\mathbb{R}^{n}}\left\|\Delta_{x} f\right\|_{p} \mu\left(|x|^{-1}\right) \frac{d x}{|x|^{n}} \\
& =C \int_{\mathbb{R}^{n}} \frac{\left\|\Delta_{x} f\right\|_{p}}{w(|x|)} \frac{d x}{|x|^{n}} .
\end{aligned}
$$

Take now $f \in B_{w, \phi}^{p, 1}$. From (2.2) in Lemma 2.1 and Fubini's theorem 


$$
\begin{aligned}
\int_{\mathbb{R}^{n}} \frac{\left\|\Delta_{x} f\right\|_{p}}{w(|x|)} \frac{d x}{|x|^{n}} & \leq C \int_{0}^{\infty}\left\|\phi_{t} * f\right\|_{p}\left[\int_{\mathbb{R}^{n}} \mu\left(|x|^{-1}\right) \min \left(1, \frac{|x|}{t}\right) \frac{d x}{|x|^{n}}\right] \frac{d t}{t} \\
& =C \int_{0}^{\infty}\left\|\phi_{t} * f\right\|_{p}\left[\int_{0}^{\infty} \mu(s) \min \left(1, \frac{1}{s t}\right) \frac{d s}{s}\right] \frac{d t}{t} \\
& =C \int_{0}^{\infty}\left\|\phi_{t} * f\right\|_{p}\left[\int_{0}^{t^{-1}} \frac{\mu(s)}{s} d s+\frac{1}{t} \int_{t^{-1}}^{\infty} \frac{\mu(s)}{s^{2}} d s\right] \frac{d t}{t} \\
& \leq C \int_{0}^{\infty}\left\|\phi_{t} * f\right\|_{p} \mu\left(t^{-1}\right) \frac{d t}{t} \\
& =C \int_{0}^{\infty} \frac{\left\|\phi_{t} * f\right\|_{p}}{w(t)} \frac{d t}{t} .
\end{aligned}
$$

Theorem 1.3. Let $1 \leq p \leq \infty, 1<q<\infty, \phi \in \mathcal{A}$ and $w$ a weight such that

$$
w(t)=\lambda^{\frac{1}{q^{\prime}}}(t) \mu^{\frac{-1}{q}}\left(t^{-1}\right)
$$

for some pair of weights $\lambda, \mu \in \mathcal{W}_{0,1}$. Then

$$
\left.\Lambda_{w}^{p, q}=B_{w, \phi}^{p, q} \quad \text { (with equivalent seminorms }\right)
$$

Proof. Assume $f \in \Lambda_{w}^{p, q}$. Let us show first that

$$
\int_{\mathbb{R}^{n}} \frac{|f(x)|}{(1+|x|)^{n+1}} d x<\infty
$$

Let us denote

$$
\Phi(x)=\frac{w(|x|)|x|^{n}}{(1+|x|)^{n+1}} .
$$

We shall see that under the assumptions $\lambda, \mu \in \mathcal{W}_{0,1}$ one has that $\Phi \in L^{q^{\prime}}\left(\mathbb{R}^{n}, \frac{d x}{|x|^{n}}\right)$. Indeed

$$
\int_{0}^{\infty} \Phi^{q^{\prime}}(t) \frac{d t}{t}=\int_{0}^{\infty} \lambda(t) \mu^{-q^{\prime} / q}\left(t^{-1}\right) \frac{t^{n q^{\prime}}}{(1+t)^{q^{\prime}(n+1)}} \frac{d t}{t} .
$$

Using (1.5) we have $\mu(s) \geq C \min (1, s)$. Therefore

$$
\begin{aligned}
\int_{0}^{\infty} \Phi^{q^{\prime}}(t) \frac{d t}{t} & \leq C \int_{0}^{\infty} \lambda(t) \max \left(1, t^{\left(q^{\prime}-1\right)}\right) \frac{t^{n q^{\prime}}}{(1+t)^{q^{\prime} n+q^{\prime}}} \frac{d t}{t} \\
& \leq C\left(\int_{0}^{1} \lambda(t) \frac{d t}{t}+\int_{1}^{\infty} \frac{\lambda(t)}{t} \frac{d t}{t}\right)<\infty
\end{aligned}
$$

From Hölder's inequality one has 


$$
\int_{\mathbb{R}^{n}} \frac{\left\|\Delta_{x} f\right\|_{p}}{(1+|x|)^{n+1}} d x=\int_{\mathbb{R}^{n}} \frac{\left\|\Delta_{x} f\right\|_{p}}{w(|x|)} \Phi(x) \frac{d x}{|x|^{n}}<\infty
$$

and we apply Lemma 2.3 .

Let us now prove

$$
\|f\|_{B_{w, \phi}^{p, q}} \leq C|| f \|_{\Lambda_{w}^{p, q}} .
$$

From (2.1) in Lemma 2.1 with $\rho=1$

$$
\frac{\left\|\phi_{t} * f\right\|_{p}}{w(t)} \leq C \int_{\mathbb{R}^{n}} K(x, t) \frac{\left\|\Delta_{x} f\right\|_{p}}{w(|x|)} \frac{d x}{|x|^{n}}
$$

where

$$
K(x, t)=\frac{w(|x|)}{w(t)} \min \left(1, \frac{t}{|x|}\right) .
$$

Take

$$
\left(\Omega_{1}, \Sigma_{1}, \mu_{1}\right)=\left(\mathbb{R}^{n}, \mathcal{B}\left(\mathbb{R}^{n}\right), \frac{d x}{|x|^{n}}\right)
$$

and

$$
\left(\Omega_{2}, \Sigma_{2}, \mu_{2}\right)=\left((0, \infty), \mathcal{B}((0, \infty)), \frac{d x}{|x|^{n}}\right) .
$$

Since $K(x, t)=R_{0,1}(|x|, t)$ we can apply Lemma 2.2 with $\varepsilon=0$ and $\delta=1$ to get a measurable function $g$ satisfying (2.3) and (2.4).

Now write $h_{1}(x)=g(|x|)$ and $h_{2}(t)=g(t)$. Obviously, using polar coordinates, (2.3) and (2.4) give (1.3) and (1.4) in Lemma B, what shows that $T_{K}$ is bounded from $L^{q}\left(\mathbb{R}^{n}, \frac{d x}{|x|^{n}}\right)$ into $L^{q}\left((0, \infty), \frac{d t}{t}\right)$. Therefore

$$
\begin{aligned}
\|f\|_{B_{w, \phi}^{p, q}} & \leq C\left\|T_{K}\left(\frac{\left\|\Delta_{x} f\right\|_{p}}{w(|x|)}\right)\right\|_{L^{q}\left((0, \infty), \frac{d t}{t}\right)} \\
& \leq C\left\|\frac{\left\|\Delta_{x} f\right\|_{p}}{w(|x|)}\right\|_{L^{q}\left(\mathbb{R}^{n}, \frac{d x}{x \mid n}\right)} \\
& \leq C\|f\|_{\Lambda_{w}^{p, q} .}
\end{aligned}
$$

(ii) Let us take $f \in B_{w, \phi}^{p, q}$. From (2.2) in Lemma 2.1

$$
\frac{\left\|\Delta_{x} f\right\|_{p}}{w(|x|)} \leq C \int_{0}^{\infty} R(x, t) \frac{\left\|\phi_{t} * f\right\|_{p}}{w(t)} \frac{d t}{t} .
$$

where

$$
R(x, t)=\frac{w(t)}{w(|x|)} \min \left(1, \frac{|x|}{t}\right) .
$$


Now take

$$
\left(\Omega_{1}, \Sigma_{1}, \mu_{1}\right)=\left((0, \infty), \mathcal{B}((0, \infty)), \frac{d x}{|x|^{n}}\right)
$$

and

$$
\left(\Omega_{2}, \Sigma_{2}, \mu_{2}\right)=\left(\mathbb{R}^{n}, \mathcal{B}\left(\mathbb{R}^{n}\right), \frac{d x}{|x|^{n}}\right)
$$

Combine now again Lemma 2.2 and Lemma $\mathrm{B}$ to get the boundedness of $T_{R}$ from $L^{q}\left((0, \infty), \frac{d t}{t}\right)$ into $L^{q}\left(\mathbb{R}^{n}, \frac{d x}{|x|^{n}}\right)$. Therefore

$$
\|f\|_{\Lambda_{w}^{p, q}} \leq C\left\|T_{R}\left(\frac{\left\|\phi_{t} * f\right\|_{p}}{w(t)}\right)\right\|_{L^{q}\left(\frac{d x}{|x|^{n}}\right)} \leq C\left\|\frac{\left\|\phi_{t} * f\right\|_{p}}{w(t)}\right\|_{L^{q}\left(\frac{d t}{t}\right)} \leq C\|f\|_{B_{w, \phi}^{p, q}} .
$$

Remark. Note that in the previous theorem one of the embedding could have been proved under weaker assumptions. In fact, if $\lambda, \mu \in \mathcal{W}_{n, 1}$ then $\Lambda_{w}^{p, q} \subseteq B_{w, \phi}^{p, q}$.

\section{REFERENCES}

[1] O. V. Besov, On a family of function spaces in connection with embeddings and extensions, Trudy Mat. Inst. Steklov 60 (1961), 42-81.

[2] O. Blasco, Operators on weighted Bergman spaces and applications, Duke Math. J. 66 (1992), 443-467.

[3] O. Blasco, G.S. de Souza, Spaces of analytic functions on the disc where the growth of $M_{p}(F, r)$ depends on a weight, J. Math. Anal. and Appl. 147 (1990), 580-598.

[4] H.-Q. Bui, Representation theoremss and atomic decompositions of Besov spaces, Math. Nachr. 132 (1987), 301-311.

[5] S. Bloom, G.S. de Souza, Atomic decomposition of generalized Lipschitz spaces, Illinois J. Math. 33 (1989), 181-189.

[6] A. P. Calderón, An atomic decomposition of distributions in parabolic $H^{p}$ spaces, Adv. in Math. 25 (1977), 216-225.

[7] A. P. Calderón A. Torchinsky, Parabolic maximal functions associated with a distribution I, Adv. in Math. 16 (1975), 1-64.

[8] R. Coifman, R. Rochberg, Representation theorems for holomorphic and harmonic functions in $L^{p}$, Astérisque $\mathbf{7 7}$ (1980), 11-66.

[9] G. S. de Souza, The atomic decomposition of Besov-Bergman-Lipschitz spaces, Proc. Amer. Math. Soc. 94 (1985), 682-686.

[10] T.M. Flett, Lipschitz spaces of functions on the circle and the disc, J. Math. Anal. and Appl. 39 (1972), 125-158.

[11] T.M. Flett, Temperatures, Bessel potentials and Lipschizt spaces, Proc. London Math. Soc. 20 (1970), 749-768.

[12] M. Frazier, B. Jawerth, Decomposition of Besov spaces, Indiana Univ. Math. J. 34 (1985), 777-799.

[13] M. Frazier, B. Jawerth, A discrete transform and decompositions of distribution spaces, J. Funct. Anal. 93 (1990), 34-170.

[14] M. Frazier, B. Jawerth, G. Weiss, Littlewood-Paley Theory and the study of function spaces, C.B.M.S. n 79 Amer. Math. Soc., Providence, Rhode Island, 1991.

[15] J. García-Cuerva, J.L. Rubio de Francia, Weighted norm inequalities and related topics, NorthHolland Math. Stud. 116, North-Holland, Amsterdam, New York, 1985.

[16] S. Janson, Generalization on Lipschitz spaces and applications to Hardy spaces and bounded mean oscillation, Duke Math. J. 47 (1980), 959-982. 
[17] S. Janson, M. Taibleson, I Teoremi di rappresentazione di Calderón, Rend. Sem. Mat. Ist. Politecn. Torino 39 (1981), 27-35.

[18] R. Rochberg, Decomposition theorems for Bergman spaces and their applications, Operators and Function Theory (S. C. Power, ed) (1985), Reidel, Dordrecht, 225-278.

[19] J. Peetre, New thoughts on Besov spaces, Duke Univ. Math. Series., Durham, NC, 1976.

[20] E. Stein, Singular integrals and differenciability properties of functions, Princeton Univ. Press, Princeton, NJ, 1970.

[21] J.O. Stromberg, A. Torchinsky, Weighted Hardy spaces, Lecture Notes in Math. 1381, Springer Verlag, Berlin, 1989.

[22] M. Taibleson, On the theory of Lipschizt spaces of distributions on euclidean $n$-space., I, II, III, J. Math. Mech. 13 (1964), 407-480; 65, 821-840; 15 (1966), 973-981.

[23] A. Torchinsky, Real variable methods in Harmonic Analysis, Academic Press, Orlando, FL, 1986.

[24] H. Triebel, Theory of function spaces, Monographs in Math., vol. 78, Birkhäuser, Verlag, Basel, 1983.

Oscar Blasco. Departamento de Análisis Matemático, Universidad de Valencia, 46100 Burjassot (VAlencia), Spain.

E-mail address: Blascod@mac.uv.es

José Luis Ansorena. Departamento de Matemáticas, Universidad de Zaragoza, 50009 ZARAGOZA, SpAIN.

E-mail address: Blasco@cc.unizar.es 